\title{
A road map for collaterals: Use of 3-dimensional techniques in tetralogy of Fallot pulmonary atresia with major aortopulmonary collateral arteries
}

From the Divisions of ${ }^{\mathrm{a}}$ Cardiology and ${ }^{\mathrm{b}}$ Cardiothoracic Surgery; and ${ }^{\mathrm{c}}$ Department of Radiology, Children's Hospital of Philadelphia, Philadelphia, Pa.

Dr O'Byrne receives support from the National Institute of Health/National Heart Lung and Blood Institute (HL130420-01). Dr Ghosh is supported by the National Institutes of Health Institutional Training Grant (T32GM008562). The funding agencies had no role in the planning or execution of the study, nor did they edit the manuscript. The manuscript represents the opinions of the authors alone.

Disclosures: Authors have nothing to disclose with regard to commercial support.

Received for publication Nov 23, 2019; revisions received Nov 23, 2019; accepted for publication Jan 3, 2020; available ahead of print Feb 12, 2020.

Address for reprints: Reena M. Ghosh, MD, Division of Cardiology, 34th and Civic Center Blvd, 8NW90 Cardiology Suite, Philadelphia, PA 19104 (E-mail: ghoshr@email.chop.edu).

JTCVS Techniques 2020;1:82-5

2666-2507

Copyright (C) 2020 The Author(s). Published by Elsevier Inc. on behalf of The American Association for Thoracic

Surgery. This is an open access article under the CC BY-NC-ND license (http://creativecommons.org/licenses/bync-nd/4.0/).

https://doi.org/10.1016/j.xjtc.2020.01.007

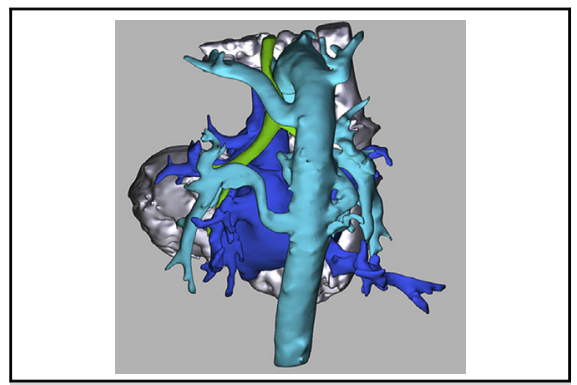

Digital reconstruction of patient anatomy illustrating the relationship of thoracic structures.

CENTRAL MESSAGE

We present an innovative 3D visualization strategy for preoperative planning in patients with tetralogy of Fallot pulmonary atresia MAPCA.

See Commentaries on pages 86 and 88.
Treatment of tetralogy of Fallot with pulmonary atresia and major aortopulmonary collateral arteries (MAPCA) remains challenging. ${ }^{1,2}$ Surgical repair involves unifocalization and arterioplasty of collaterals and frequently diminutive pulmonary arteries (PAs). ${ }^{2}$ These vessels are frequently torturous and complex, limiting the utility of traditional imaging modalities (eg, echocardiography and angiography). ${ }^{3}$ We present images from a novel visualization strategy to enhance preoperative understanding of the anatomy.

Three patients at the Children's Hospital of Philadelphia underwent unifocalization and tetralogy repair from December 2018 to February 2019. Per institutional protocol, all patients underwent cardiac computed tomography angiography in the neonatal period, with subsequent echocardiograms and cardiac catheterization for presurgical planning (Figure 1). Before surgery, a digital 3-dimensional (3D) reconstruction of the thoracic anatomy was created with the use of the computed tomography angiography images (Materialise Mimics and 3-Matics Design, Leuven, Belgium).

Patient 1 had an absence of confluent PAs, 5 MAPCAs with distinct aortic origins (Figure 2), a right aortic arch with an aberrant origin of the left subclavian artery, and no evidence of a patent ductus arteriosus. Patient 2 had confluent branch PAs, 4 MAPCAs arising from the proximal descending aorta, and a left aortic arch (Figure 3). Patient 3 had hypoplastic confluent branch PAs, 4 MAPCAs with 3 distinct origins from the proximal descending aorta, and a left aortic arch (Figure 4).

The digital reconstructions subjectively increased comprehension of the spatial relationship of the collateral vessels to the native PAs, airway, and pulmonary veins for the surgical team. Presented in a 3D PDF format, the file's interactive nature allowed the user to virtually "turn on and off" any of the surrounding structures (Video 1). Intraoperatively, the surgeon confirmed the fidelity of the reconstructions. This $3 \mathrm{D}$ visualization strategy was quickly adopted by the cardiothoracic surgical group and is now part of routine presurgical planning for this patient population.

\section{References}

1. Bull K, Somerville J, Ty E, Spiegelhalter D. Presentation and attrition in complex pulmonary atresia. J Am Coll Cardiol. 1995;25:491-9.

2. Bauser-Heaton H, Borquez A, Han B, Ladd M, Asija R, Downey L, et al. Programmatic approach to management of tetralogy of Fallot with major aortopulmonary collateral arteries: a 15-year experience with 458 patients. Circ Cardiovasc Interv. 2017; 10:102.

3. Anwar S, Rockefeller T, Raptis DA, Woodard PK, Eghtesady P. 3D printing provides a precise approach in the treatment of tetralogy of Fallot, pulmonary atresia with major aortopulmonary collateral arteries. Curr Treat Options Cardiovasc Med. 2018;20:5. 

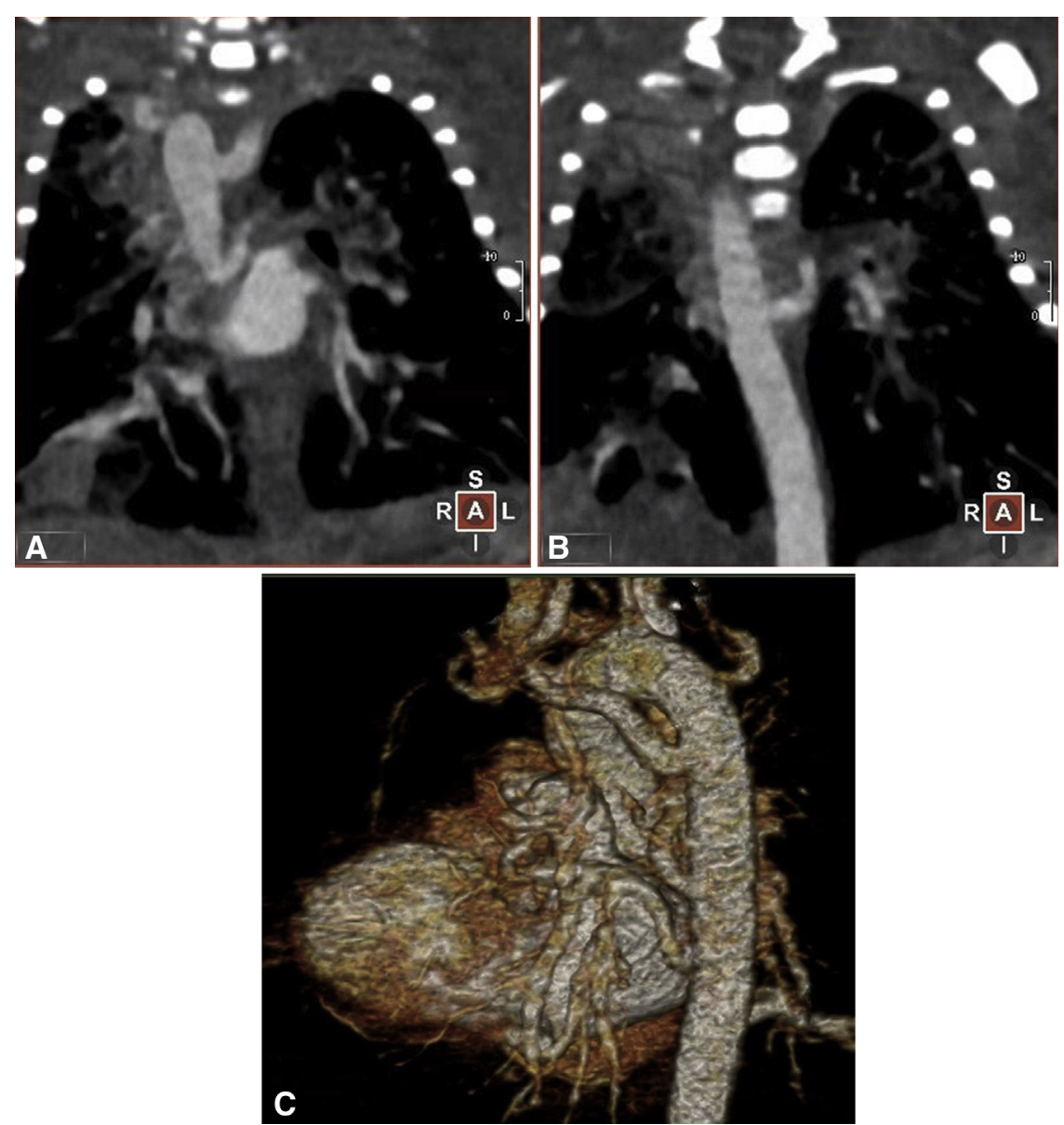

FIGURE 1. Chest computed tomography angiography of a patient with tetralogy of Fallot pulmonary atresia MAPCA. A and B, Two different coronal slices from the same computed tomography angiography demonstrating MAPCAs arising from the descending aorta. C, Volume rendering of the patient's blood pool.
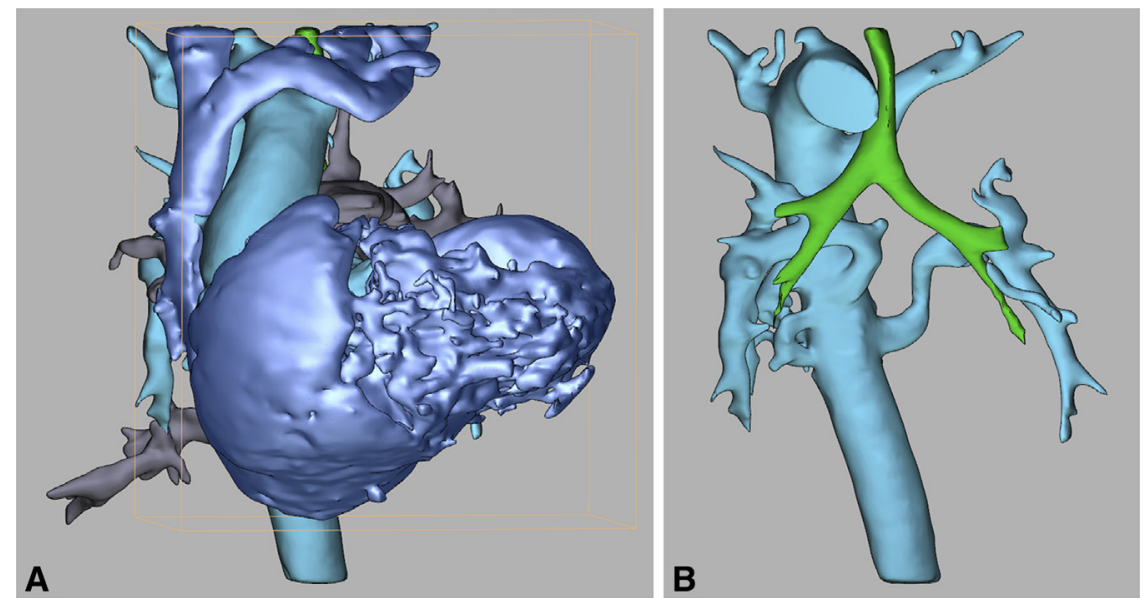

FIGURE 2. Digital 3D PDF of patient 1's anatomy (tetralogy of Fallot pulmonary atresia and absence of mediastinal PAs and 5 collateral vessels arising from the descending aorta). A, Anteroposterior view with slight caudal rotation. B, Same view with heart and pulmonary veins removed. Blue: superior vena cava, innominate vein, and cardiac mass. Turquoise: aorta and MAPCAs. Grey: pulmonary veins. Green: trachea and mainstem bronchi. 


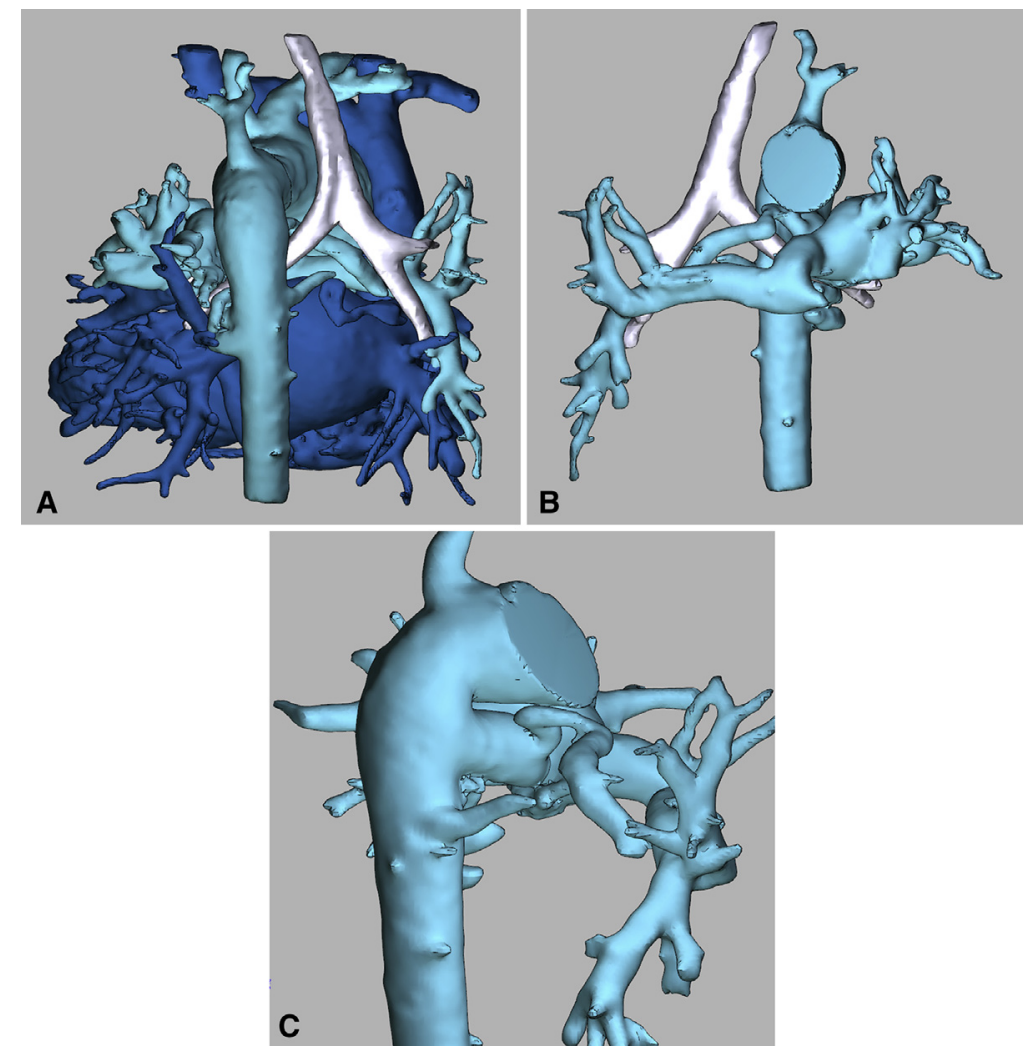

FIGURE 3. Digital 3D PDF of patient 2's anatomy (tetralogy of Fallot pulmonary atresia and confluent branch PAs, with 4 collateral vessels arising from the descending aorta). A, Posterior view. B, Anterior view with heart removed. C, Posterior view with lateral rotation. Blue: superior vena cava, innominate vein, cardiac mass including pulmonary veins. Turquoise: aorta, MAPCAs, and confluent branch PAs. Silver: trachea and mainstem bronchi. 

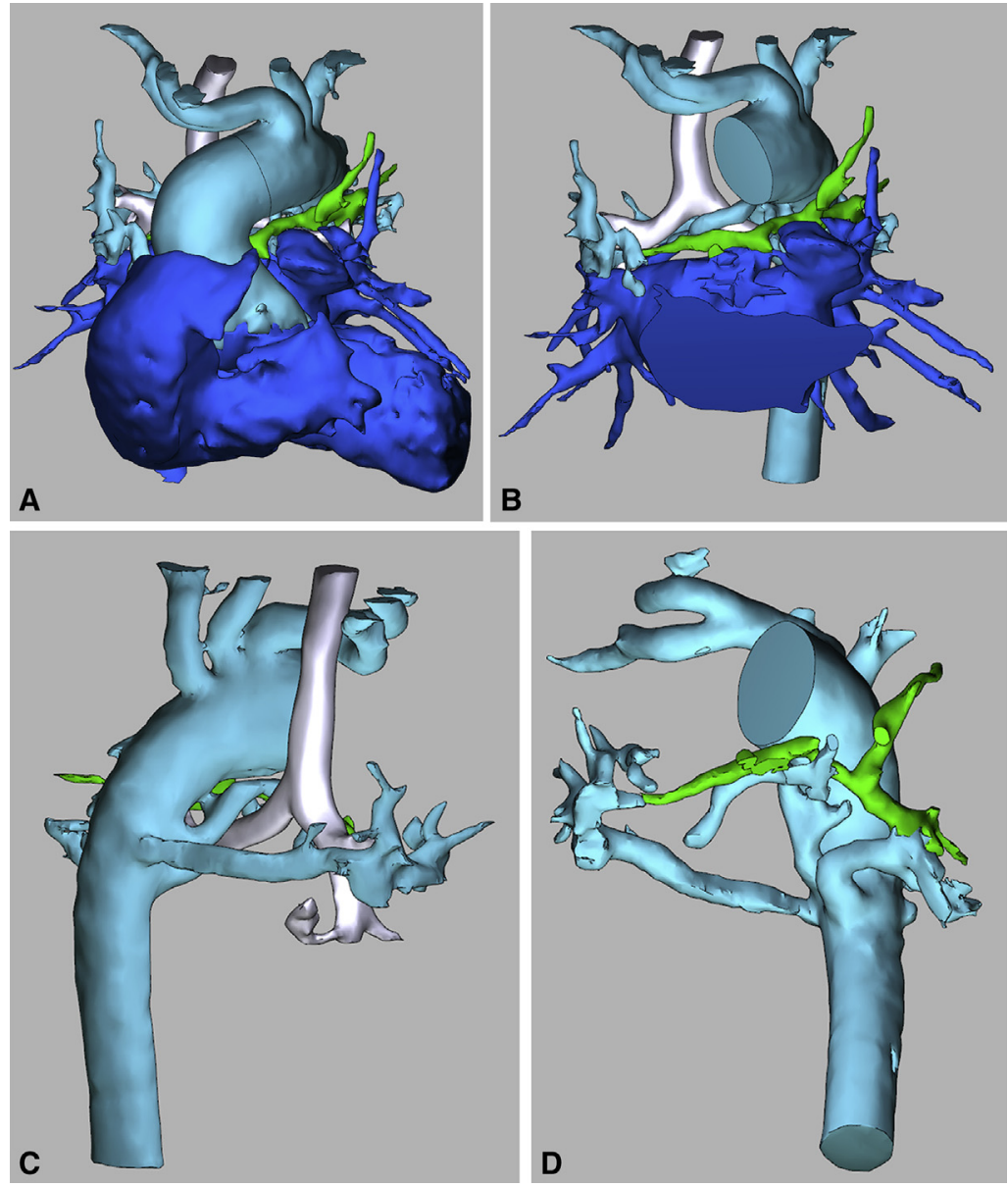

FIGURE 4. Digital 3D PDF of patient 3's anatomy (tetralogy of Fallot pulmonary atresia with hypoplastic confluent branch PAs and 4 collateral vessels with 3 distinct origins from the proximal descending aorta). A, Anterior view. B, Anterior view with anterior cardiac mass remove. C, Posterior view with lateral rotation. D, Anterior view with slight caudal rotation. Blue: cardiac mass. Turquoise: aorta and MAPCAs. Green: confluent branch PAs. Silver: trachea and mainstem bronchi.

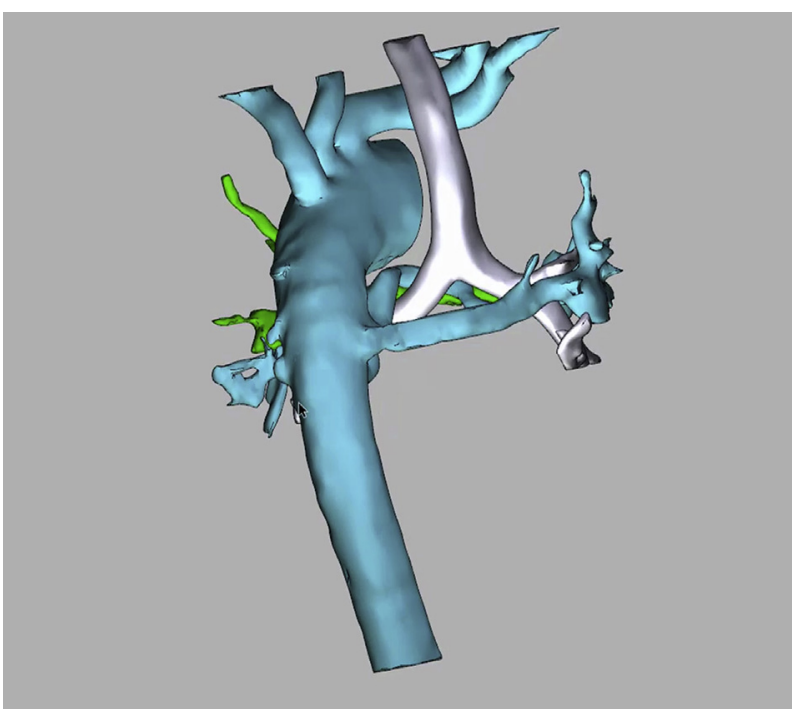

VIDEO 1. On-screen recording of 3D PDF manipulation of patient 3's anatomic reconstruction. Blue: cardiac mass. Turquoise: aorta and MAPCAs. Green: confluent branch pulmonary arteries. Silver: trachea and mainstem bronchi. Video available at: https://www.jtcvs.org/article/S2666-2507(20) 30024-9/fulltext. 